\title{
Using Advances in Neuroimaging to Detect, Understand, and Monitor Disease Progression in Huntington's Disease
}

\author{
H.D. Rosas, ${ }^{* I l}$ A.S. Feigin, ${ }^{\dagger}$ and Steven M. Hersch ${ }^{\S}$ \\ *Center for Neuroimaging of Aging and Neurodegenerative Diseases, Massachusetts General Hospital (MGH), Charlestown, \\ Massachusetts 02129; ${ }^{\dagger}$ Center for Neurosciences, North Shore Long Island Jewish Research Institute, and the Department of \\ Neurology, North Shore University Hospital, Manhasset, New York 11030; ${ }^{\ddagger}$ Department of Neurology, New York University \\ School of Medicine, New York, New York 10016; ${ }^{\S}$ Massachusetts General Institute for Neurodegenerative Disease, MGH, \\ Charlestown, Massachusetts 02129; "IAthinoula A. Martinos Center for Biomedical Imaging, MGH/Harvard Medical School, \\ Charlestown, Massachusetts 02129
}

\begin{abstract}
Summary: Trangenic mouse models and other screens are being used to identify potential therapeutic agents for use in clinical trials in Huntington's disease (HD). The development of surrogate markers that can be used in clinical therapeutics is an active area of research. Because HD is relatively uncommon and only a portion of available subjects meet inclusion and exclusion criteria, therapeutic trials are limited by the availability of potential subjects as well as the relative insensitivity of the clinical measures used. Neuroimaging methods offer the
\end{abstract}

potential to provide noninvasive, reproducible, and objective methods not only to better understand the disease process but also to follow in clinical studies to determine if a drug is effective in slowing down disease progression or perhaps even in delaying onset. Following is a review of the literature, which highlights the studies that have been published to date. Key Words: Huntington's disease, neuroimaging, MRI, PET, biomarkers, MRS.

\section{INTRODUCTION}

New advances in modern neuroimaging techniques are enabling scientists to study the progressive structural, physiological, and functional changes that occur in Huntington's disease (HD) and how these changes may explain behavioral, cognitive, and motor symptoms. Perhaps more importantly, these noninvasive methods may provide objective, quantitative, and reliable measures that may be helpful in determining the onset of HD and in measuring its progression. As such, they may provide important surrogate markers and have utility in the assessment of potential neuroprotective interventions to retard the onset of symptoms in individuals at risk for HD or slow progression in those already affected. In addition, because the genetic test for HD enables the identification of individuals fated to develop symptoms, neuroimaging provides a unique opportunity to study individuals in the motor "presymptomatic" period for

Address correspondence and reprint requests to H. Diana Rosas, Center for Neuroimaging of Aging and Neurodegenerative Diseases, 149 13th Street, Room 2273, Charlestown, MA 02129. E-mail: rosas@helix.mgh.harvard.edu. whom neuroprotective strategies may have a particularly important role before brain pathology becomes clinically manifest.

\section{BACKGROUND}

HD is an autosomal dominant inherited neurodegenerative disorder that is characterized by progressive motor dysfunction, emotional disturbances, and dementia, and inexorably leads to death. The average age of onset is $\sim 37$ years of age; however, the range of onset is from infancy into the 80s. Affected individuals are rapidly disabled by early functional decline and require care and supervision for another 15-25 years before succumbing to the effects of severe physical and mental deterioration. HD occurs worldwide, in all races and ethnic groups. ${ }^{1}$ Its prevalence is $5-10$ cases per 100,000 , and there is a new mutation rate as high as $1-3 \% .^{2,3}$ There are about 30,000 affected individuals in the United States. Another 150,000 Americans have a genetic risk for developing the disease; however, for a variety of well understood reasons less than $4 \%$ of individuals at risk who are 18 years of age or older have undergone genetic testing for 
the HD gene in the United States. ${ }^{4}$ There is no therapy proven to delay onset or to slow progression, and current medical care focuses on managing symptoms and optimizing function. There are increasing reasons to believe that pathologic alterations occur in the brain for years before symptoms manifest. The HD gene, first cloned in $1993,{ }^{5}$ codes for a large, highly conserved protein of unknown function ("huntingtin") containing 3144 amino acids. In individuals with $\mathrm{HD}$, a polymorphic trinucleotide repeat sequence (CAGn) near the 5 ' end of the gene is expanded beyond the normal repeat range, ${ }^{5}$ leading to translation of an expanded polyglutamine sequence in the protein. In the normal population, the number of CAG repeats varies from 17 to 29. In individuals with HD there are more than 38 repeats. Once expanded into the pathogenic ranges, there is an inverse relationship between the CAG repeat number and the age of onset; however, there is not a strong relationship between CAG repeat length and the progression of illness when signs and symptoms of disease have become manifest. ${ }^{6,7}$

The hallmark of neuropathology in HD is selective neurodegeneration in which vulnerable populations of neurons degenerate whereas less vulnerable populations are spared. ${ }^{77}$ The earliest and most striking neuropathologic changes are thought to occur in the neostriatum, but neuronal loss has been identified in many other regions of the brain. ${ }^{77}$

There is no proven therapy to delay onset or slow progression of disease. However, new understanding about the mechanisms of disease is being acquired at a rapid rate and neuroprotective compounds are emerging from preclinical studies that are already making their way into clinical trials.

\section{Pathophysiology of HD}

Although many leads have been uncovered in the last decade, a direct pathway from the genetic mutation to neuronal dysfunction and death has not yet been established. Huntingtin is a widely expressed, predominantly cytoplasmic protein of unknown function found heterogeneously in neurons throughout the brain. ${ }^{10-13}$ In HD, both normal and mutant alleles are expressed and both gain-of-function alterations in which mutant huntingtin is toxic and loss-of-function alterations ${ }^{14}$ in which suppression of normal huntingtin functions might also be toxic have been identified. The proteolysis of mutant huntingtin into a toxic $\mathrm{N}$-terminal fragment ${ }^{16}$ has been shown in humans and in animal and cellular models to lead to the formation of protein aggregates in the nucleus, cytoplasm, and processes of neurons..$^{10,17,18}$ These aggregates can be readily detected in the brains of individuals at risk for HD who died before exhibiting any symptoms. ${ }^{18} \mathrm{In}$ transgenic models, aggregates concentrate in the cortex, in which their density correlates with the number of CAG repeats. ${ }^{19}$

\section{Experimental therapeutics in HD: the preclinical pipeline}

The primary focus of experimental therapeutics is pharmacological neuroprotection, preventing the dysfunction and death of CNS neurons that underlie HD. Specific neuroprotective strategies targeted at identified molecular mechanisms have the potential to dramatically delay the onset and slow the progression of HD. Since the neurodegenerative process in the brain begins before symptoms of HD are recognizable, neuroprotective strategies should optimally be started before the onset of symptoms in individuals with proven genetic risk. There are increasing reasons to believe that there are many dysfunctional neurons present at any given time in the course of HD. As was recently modeled in a conditional HD transgenic mouse, affected individuals have a population of injured neurons that may be able to recover, providing there is some reversibility during both the presymptomatic and early symptomatic periods.

Recent studies in HD transgenic mice using glutamate antagonists such as remacemide, drugs that enhance mitochondrial function, such as coenzyme Q10 and creatine ${ }^{20}$ dichloracetate, ${ }^{21}$ lipoic acid, ${ }^{22}$ the antiapoptotic antibiotic minocycline, ${ }^{23}$ the transglutaminase inhibitor cystamine, ${ }^{24}$ and several transcriptional modulators ${ }^{25}$ have demonstrated significant neuroprotective effects. The range of effects on survival has been about 8-29\% and a number of these candidate therapies are already being studied in early phase clinical trials. These compounds represent the immediate pipeline of neuroprotective agents, whereas a number of medium-throughput and high-throughput screening efforts are identifying many additional leads that will be tested in HD transgenic mice in the coming years.

\section{The need for biomarkers}

Traditionally, the clinical diagnosis of HD has relied upon the development of abnormal motor signs in a person at risk for HD by virtue of having an affected parent. Standard clinical instruments are often used to help define symptoms of HD and their progression, most commonly the Unified Huntington's Disease Rating Scale (UHDRS), ${ }^{5,26}$ which was developed and validated by Huntington Study Group (HSG). The UHDRS, which has become the standard instrument for following the progression of symptomatic HD, assesses four major domains: (1) motor, (2) cognitive, (3) behavioral, and (4) functional capacity. The UHDRS has been used in many clinical trials and is a primary measure in all on-going clinical trials by the HSG and by other groups performing HD studies. It is used in asymptomatic at-risk individuals to detect the onset of symptoms and in symptomatic individuals to follow their progression. The total functional capacity subscale (TFC) has been the most stable and reliable of the clinical measures that are in- 


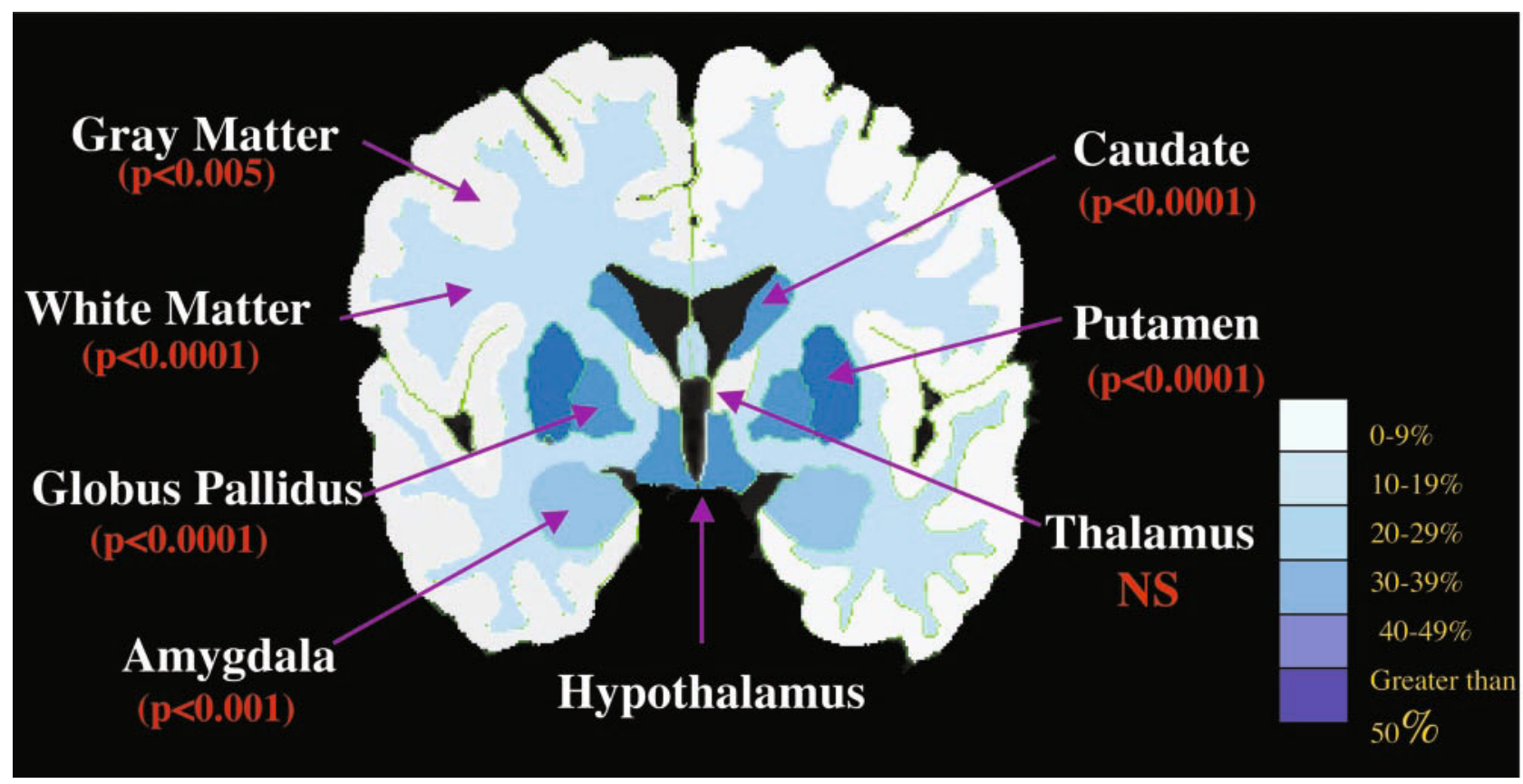

FIG. 1. Results of the manual segmentation of $18 \mathrm{HD}$ subjects in early stages of disease. Almost all regional brain volumes were statistically smaller than healthy age-matched controls. Percentage volume loss was similar to values reported in neuropathological studies.

cluded in the UHDRS. However, the symptoms and progression of $\mathrm{HD}$ are highly variable and these clinical measures are subject to some degree of inter-rater and intrarater variability. Trials of neuroprotective treatments for $\mathrm{HD}$ thus require large numbers of patients and long trial durations. Given the growing pipeline of potential neuroprotective agents attractive for testing in clinical trials there is a crucial need to develop valid, reliable, and reproducible surrogate markers of both disease onset and disease progression using newer neuroimaging technologies.

\section{MAGNETIC RESONANCE IMAGING}

\section{Morphometric analyses}

Basal ganglia pathology has been the focus of much work using magnetic resonance imaging (MRI) methods to quantitate brain volumes and has been central to the development of hypotheses about possible mechanisms of death. Alexander et al. ${ }^{27}$ proposed the existence of five distinct parallel segregated loops connecting the basal ganglia to distinct cortical regions and it has been hypothesized that disruption of these loops at the level of the basal ganglia could result in the motor, cognitive, and neuropsychiatric symptoms of disease. As a result, early imaging studies focused primarily on the study of the striatal volumetric changes. Several studies demonstrated evidence of basal ganglia atrophy, ${ }^{28,29}$ even as early as 7 years before the onset of motor symptoms. ${ }^{30}$ Caudate volumes were found to correlate with performance on neuropsychological tests, suggesting that caudate atrophy may play a role in cognitive symptoms. ${ }^{31} \mathrm{~A}$ mea- surable rate of change in caudate volume over time was reported, ${ }^{28,30}$ which correlated with age at onset and length of trinucleotide repeat, suggesting that striatal volume loss is a potentially important surrogate marker of HD that may be useful in clinical therapeutic trials.

Another morphometric study of patients in early stages of disease ${ }^{32}$ found statistically smaller volumes of the striatum (putamen, caudate, and pallidum) and several other brain structures, including hippocampus, pallidum, brainstem, cerebral gray and white matter, as compared to age-matched healthy control subjects (FIG. 1). This suggests that more widespread atrophy is present even in early stages of disease. Because volume loss of these structures correlated with clinical measures, it is likely that both striatal and extrastriatal degeneration play a role in the clinical expression of HD.

These morphometric studies used manual segmentation methods, which are time and effort intensive and which depend on a rater's skill and bias. As such, only relatively small numbers of subjects were studied. Emerging semi-automated morphometric analytical tools have been developed that are providing faster, more reliable, and more reproducible methods. ${ }^{33}$ Voxel-based morphometry (VBM) is a fully automated method that detects regional structural changes on a voxel-wise basis between groups of subjects. ${ }^{34}$ This is achieved by various preprocessing steps, which include extraction of the whole brain, spatial normalization into a stereotactic space, segmentation into gray matter, white matter and CSF compartments, modulation for volume changes 
caused by spatial normalization and finally, smoothing using a Gaussian kernal. ${ }^{34}$

One study ${ }^{35}$ used this method to study a group of gene-positive presymptomatic individuals. They found reductions in the gray matter volume of the striatum, amygdala, insula, dorsal midbrain, and bilateral intraparietal sulcus in the gene-positive group as compared to the healthy control group as well as significant reductions of the periventricular white matter volume. Analyses using VBM, however, can be confounded by systematic registration errors during the spatial normalization and therefore data thus analyzed should be interpreted with caution.

Another study used a novel surface reconstruction technique to measure the thickness of the cortex in 13 subjects with HD. ${ }^{36}$ This method generates an automated estimate of the gray-white boundary, which is used to determine the mean cortical thickness of individual subjects. The surface is then deformed into a canonical surface that maintains the natural topology of the brain and aligns subjects with a high resolution to compare among subjects. This method is particularly robust and is less prone to inaccuracies due to partial volume averaging and bias field inhomogeneities of the magnetic field across the brain, for example, both of which may influence voxel-based morphometric methods.

Investigation found early, regionally specific degeneration of the cortex, primarily in primary sensorimotor and higher-order association cortices. Patients in earlier stages of disease demonstrated more prominent thinning of posterior cortical regions; with disease progression, more anterior cortical regions were affected, mimicking the gradient of progressive basal ganglia pathology that has been described. Studies of individuals who are genepositive but not yet symptomatic are currently underway. Preliminary results suggest that cortical thinning is present in these individuals (personal observations), suggesting that cortical degeneration may play a role in the clinical symptoms.

\section{Summary of morphometric analyses}

Recent neuroimaging studies are redirecting attention away from the striatum to other brain regions and suggest that more generalized brain atrophy occurs in earlier stages of disease. The relative roles of the cerebral cortex and basal ganglia in psychiatric, behavioral, and neuropsychiatric symptoms of HD are still poorly understood, although there is increasing evidence that early cortical degeneration may be responsible for some of these symptoms. Because the entire cerebral cortex projects to the striatum, it may be difficult to separate the relative roles of the cortex or the striatum or to determine a cause and effect. Nevertheless, this is of particular importance in clinical therapeutic trials. If, for example, cortical atrophy contributes to clinical symptoms, then treatments targeted at preserving the striatum but not the cortex may not have any effect on the clinical symptoms. In addition, it is likely that caudate atrophy becomes asymptotic in the middle stages of disease, therefore following the atrophy of the entire brain may provide a better biomarker of progression.

Further work is needed to determine the precise relationship between clinical symptoms and regional volume changes and may provide an important surrogate marker of disease onset and/or disease progression. Newer automated segmentation methods have been developed that will enable the study of large groups of patients. As such, morphometric studies may be of great value in the design and interpretation of future clinical neuroprotective trials.

\section{FUNCTIONAL IMAGING IN HD: PET AND FMRI}

Functional neuroimaging with positron emission tomography (PET) and functional MRI (fMRI) may play several roles in the study of HD. These imaging modalities may be useful for investigating the physiological underpinnings of the behavioral, cognitive, and motor changes of the disease and many studies of symptomatic individuals using these neuroimaging modalities have demonstrated alterations in activation.

It has been hypothesized that neurons may be at risk for some time before they die, and symptoms may reflect neuronal dysfunction rather than neuronal loss. Similarly, there is growing evidence that many cognitive difficulties may be present long before motor symptoms. ${ }^{37,38}$ Early cognitive dysfunction in HD has been described in models of spatial recognition/working memory, ${ }^{39,40}$ planning, ${ }^{40}$ visual, response time, ${ }^{41}$ set shifting, and visuoperceptual and visuospatial functioning. ${ }^{40,42}$ Thus, it is possible that functional neuroimaging modalities may be more sensitive to the earliest changes that occur in disease than structural studies are. Therefore, it is possible that functional neuroimaging studies such as fMRI and PET may be of greatest utility in the assessment of potential neuroprotective interventions to retard the onset of symptoms in individuals at risk for HD.

\section{fMRI}

fMRI allows mapping of hemodynamic changes in the brain noninvasively and relies on a natural change in the magnetic properties of the blood during neuronal activity. It has emerged as an important tool in cognitive neuroscience, which has only recently been used to explore the functional alterations that occur in HD. Reduced fMRI signal was observed in symptomatic patients relative to controls in occipital, parietal, and somatomotor cortex and in the caudate, whereas increased signal was found in HD in the left postcentral and right middle 


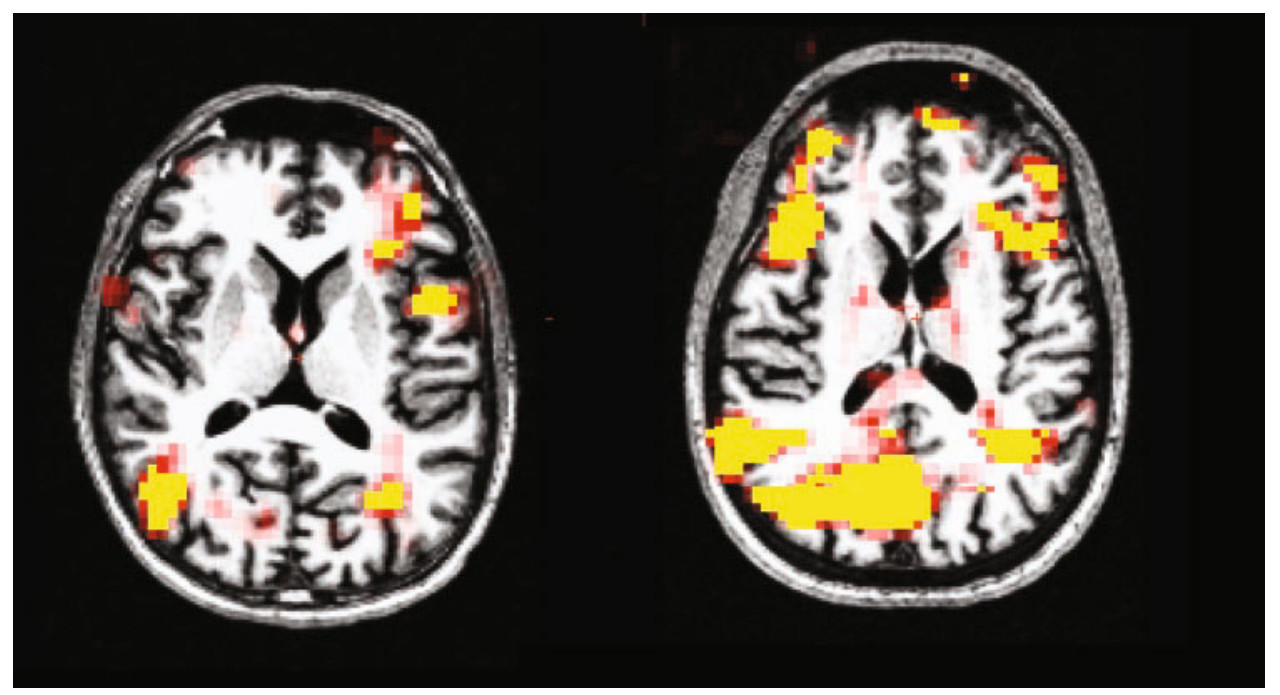

FIG. 2. fMRI study using a working memory task. Left: Control subject. Right: Presymptomatic subject. Task performance was comparable between the two subjects, but the presymptomatic subject recruited additional cortical regions to perform the task.

frontal gyri in a pilot study of three individuals with HD during performance on a Porteus maze task. ${ }^{43}$

In another study, ${ }^{44}$ modulation of the blood oxygen level-dependent (BOLD) MRI signal was found in the caudate and thalamus during a masked prime task, suggesting a role for the caudate and thalamus in altered motor control in HD. There are no published studies of presymptomatic individuals but our own preliminary data, obtained using a working memory task (FIG. 2), suggests that these individuals recruit additional cortical regions to perform the task, suggesting a compensatory reallocation of cognitive resources. When taken together, these studies suggest that for a period of time, perhaps when neurons are dysfunctional, individuals at risk for HD are able to recruit other cortical and subcortical regions to compensate. However, as neurons degenerate, these individuals are no longer able to sufficiently use compensatory mechanisms, resulting in the loss of BOLD signal and poor performance on the respective task. If this were the case, it suggests that early neuronal dysfunction may be detected with fMRI in the absence of measurable atrophy and may be a more sensitive measure of disease onset. As such, fMRI may be an important tool in future clinical neuroprotective studies when the endpoint is delay of disease onset. Additional work is necessary to determine if fMRI is useful in already symptomatic individuals to determine if a therapy has a functional benefit.

\section{PET imaging in HD}

Several investigators have used PET to examine the neurophysiological bases for cognitive impairment in HD. Two PET ligands have been predominantly used to investigate this abnormality: $\left[{ }^{11} \mathrm{C}\right]$-raclopride (RAC) as a measure of postsynaptic dopamine $\mathrm{D}_{2}$ receptor binding, and $\mathrm{H}_{2}{ }^{15} \mathrm{O}$ as a measure of cerebral blood flow. Some studies have correlated resting-state RAC binding with performance on neuropsychological tests, whereas others have measured brain activation patterns during the performance of motor sequence learning tasks.

Both striatal and extrastriatal RAC binding have been found to correlate with aspects of cognitive function in symptomatic and presymptomatic HD. Caudate RAC binding in HD correlates with performance on several timed neuropsychological tests, including the symboldigit modalities test, the Stroop, and the trail-making test, whereas severity of bradykinesia and other motor features of HD correlate with putamenal RAC binding. ${ }^{45}$ Several earlier studies had found similar correlations using both $\mathrm{RAC}^{46}$ and the PET dopamine transporter ligand $\left[{ }^{11} \mathrm{C}\right] \beta$-CIT. ${ }^{47}$ Even in presymptomatic carriers of the HD mutation, performance on multiple neuropsychological tests has been observed to correlate with striatal (putamen and caudate) RAC binding, ${ }^{48}$ and progressive declines in striatal RAC binding correlate with deterioration in neuropsychological performance on some frontal executive tasks. ${ }^{49}$ Extrastriatal reductions in RAC binding have also been observed in HD in amygdala, frontal cortex, and temporal cortex, regions known to be involved with emotional and cognitive function. ${ }^{49}$ Taken together, these observations support the idea that the cognitive effects of HD are likely mediated through a combination of direct cortical dysfunction and disruption of cognitive circuits involving corticostriatal connections.

Motor sequence learning is impaired in manifest and presymptomatic $\mathrm{HD}^{20,50}$ PET scanning with $\mathrm{H}_{2^{15}} \mathrm{O}$ during the performance of a motor sequence learning task reveals that presymptomatic HD subjects and agematched healthy controls both activate dorsolateral prefrontal cortex (DLPFC), premotor cortex, and parietal 

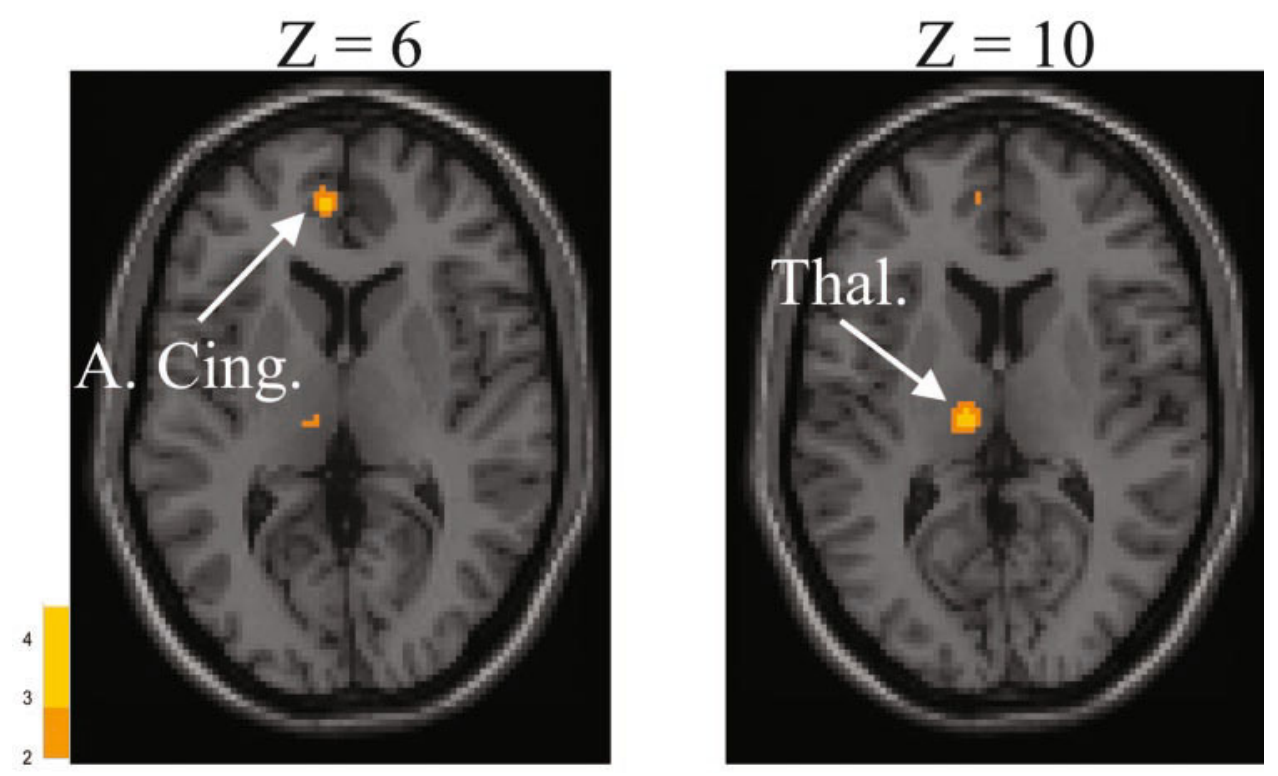

FIG. 3. $\mathrm{H}_{2}{ }_{15} \mathrm{O} / \mathrm{PET}$ brain activation responses during a motor sequence learning task in presymptomatic HD. Presymptomatic HD subjects activate the anterior cingulate (A. Cing.) and thalamus (Thal.) more than age-matched controls during learning. ${ }^{5}$

areas to perform the task. The presymptomatic HD subjects, however, unlike the controls, also activate anterior cingulate and mediodorsal thalamus (FIG. 3). ${ }^{51}$ In the same subjects, these investigators found that baseline decrements in learning and impaired activation of DLPFC during task performance correlated with reductions in caudate RAC binding. ${ }^{52}$ Furthermore, when subjects returned for follow-up 18 months later (still presymptomatic), declines in striatal RAC binding no longer correlated with learning-related activation of DLPFC but correlated with activation increases on $\mathrm{H}_{2}{ }_{15} \mathrm{O} / \mathrm{PET}$ in the hippocampus. These observations suggest that as striatal dysfunction occurs, subjects have difficulty normally accessing the DFPLC, and the brain attempts to compensate by activating cingulate, thalamic, and hippocampal structures. Ultimately, these compensatory strategies fail, and performance declines as patients enter the symptomatic phase of the illness.

\section{Cross-sectional PET studies}

PET has been used to identify resting-state functional abnormalities in the brains of HD gene carriers both preclinically and following the onset of symptoms. $\left[{ }^{18} \mathrm{~F}\right]-$ fluorodeoxyglucose (FDG) PET studies in symptomatic HD patients have consistently demonstrated reduced striatal metabolic rates. ${ }^{53-56}$ By contrast, FDG/PET data in presymptomatic individuals at risk for $\mathrm{HD}$ have been inconsistent, with some studies demonstrating normal metabolic rates ${ }^{57}$ and others demonstrating reduced levels of striatal glucose metabolism. ${ }^{58-61}$ In a PET investigation of preclinical and early-stage HD, a brain metabolic pattern relating to gene carrier status independent of symptoms was identified through the application of a form of principal component analysis (network analysis) of FDG/PET data from preclinical gene carriers (with normal RAC binding) and gene-negative control subjects. ${ }^{62}$ This HD-related pattern (HDRP) of regional brain metabolism was characterized by relative hypometabolism in the caudate and lentiform nuclei and the mesial temporal cortex, covarying with metabolic increases in occipital cortex. This study has now been reproduced and validated in a second cohort of presymptomatic HD gene carriers. ${ }^{62}$ In a cross-sectional study, individual subject expression of the HDRP is increased in presymptomatic subjects with abnormal reductions in RAC binding and further increased in symptomatic HD patients, ${ }^{62}$ suggesting that this may be a useful marker for following both presymptomatic and symptomatic HD.

Similar to FDG studies, PET studies with RAC have observed reduced striatal $\mathrm{D}_{2}$ binding in symptomatic $\mathrm{HD}$ patients, ${ }^{58,63}$ but not consistently in the presymptomatic phase of the illness. ${ }^{58,64}$ Nonetheless, CAG repeat length has been found to correlate with age-adjusted reductions in striatal RAC binding in both presymptomatic and symptomatic HD. ${ }^{65}$

\section{Longitudinal progression studies}

Several investigators have examined longitudinal changes in PET measures in small cohorts of HD patients. One group found $\sim 5 \%$ per year decline in RAC bonding in 12 symptomatic HD subjects. ${ }^{49}$ Baseline striatal RAC binding correlated with clinical scores on the UHDRS, but changes in these measures over time did not correlate. Two other small longitudinal studies found similar rates of decline. ${ }^{66,67}$ Another study measured the change in expression of the HDRP over 18 months in seven presymptomatic HD gene carriers and correlated 

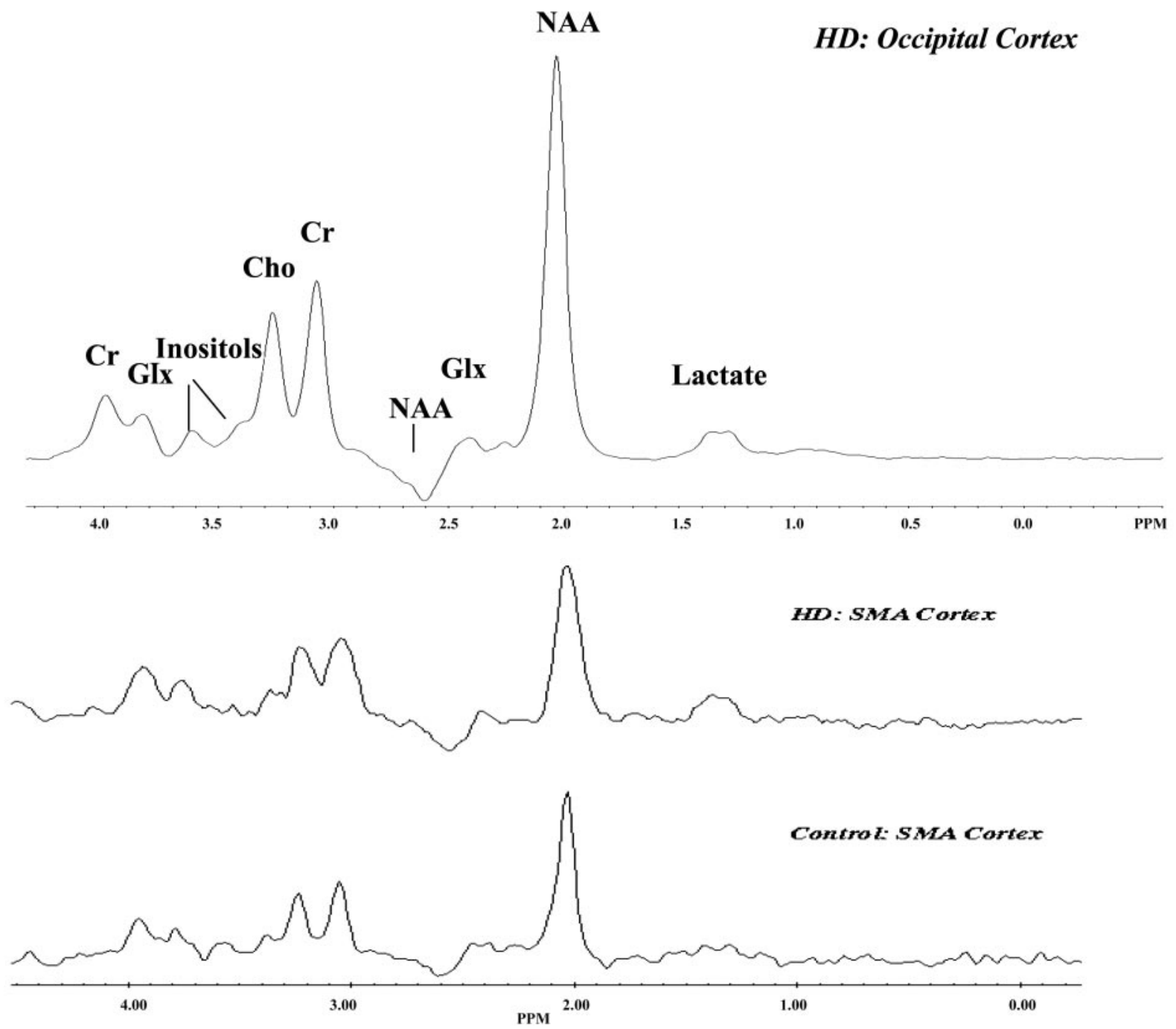

FIG. 4. Magnetic resonance spectroscopy. The occipital cortex spectra is the sum of spectra from 38 subjects with HD. Note the elevation of the lactate peak. $\mathrm{Cr}=$ creatine; $\mathrm{Glx}=$ glutamate, glutamine; Cho = choline.

these changes with declines in dopamine $\mathrm{D}_{2}$ receptor binding. ${ }^{52}$ They found that RAC binding declined by $3.9 \%$ per year in caudate, and by $4.1 \%$ per year in putamen, and that HDRP subject scores increased by a mean of 4.1 points over the period of follow-up. Baseline HDRP subject scores correlated with baseline striatal RAC binding though changes in these measures did not correlate.

\section{Summary of PET and fMRI}

The studies reviewed above support the idea that PET may be useful for measuring the pathological progression of HD. However, the contribution of brain atrophy to the changes seen with PET imaging in HD has been inadequately studied, and for this reason it remains uncertain whether PET will provide information regarding HD progression beyond what can be achieved with MRI. Nonetheless, PET studies will continue to provide illuminating information regarding the changes in brain function that underlie the various clinical manifestations of HD. In the future, PET imaging may play a role in the evaluation of symptomatic therapies for this devastating disorder.

\section{MAGNETIC RESONANCE SPECTROSCOPY}

Neurochemical alterations in the cerebral cortex in HD have been identified in neuropathological studies. For example glutamate, the neurotransmitter of pyramidal cells and excitatory inputs from the cortex to striatum, has been found in higher concentrations in cortex. ${ }^{68}$ In contrast GABA, a marker for inhibitory cortical interneurons, has been reported as reduced in cerebral cortex in HD. ${ }^{69}$ Magnetic resonance spectroscopy (MRS) has been used to measure a number of neurochemical alterations in HD in vivo (FIG. 4). 1H MRS has been useful in identifying alterations in several markers of oxidative stress. Lactate is the end product of glycolysis and ac- 
cumulates when oxidative metabolism cannot meet energy requirements. Lactate concentrations have been found to be as much as four-fold higher in the striatum of individuals with HD in some studies, ${ }^{70,71}$ although one study did not find elevated lactate concentrations. ${ }^{72}$ The lactate peak is small and may overlap with the lipid peak at short echo times; therefore, it is a difficult biomarker to follow.

Higher concentrations of choline, which may represent neuronal membrane breakdown and the liberation of glycerolphosphocholine, have been found in the striatum and frontal regions. Similarly, decreased levels of Nacetylasparate (NAA), a marker of neurons, ${ }^{71,73}$ have been found in striatum, likely reflecting neuronal loss or possibly of impaired mitochondrial energy production. Levels of NAA have been shown to progressively decline with progression of disease in human studies and have been found in the absence of neuronal loss in transgenic mouse studies. ${ }^{74}$ Attenuation of expected NAA reductions were used as a marker of neuroprotection using creatine in a transgenic mouse model. ${ }^{20}$ Creatine levels have also been found to be reduced in some studies, even in gene-positive presymptomatic individuals, and have been found to correlate with the motor score of the UHDRS and with computed measurements of saccadic and tapping speed. ${ }^{73}$ Increases in brain concentrations of creatine using creatine supplementation have been reported in transgenic mouse models as well. ${ }^{74}$ The major disadvantage of MRS is its relative insensitivity; molecules that occur in small amounts $(<0.1 \mathrm{~mm})$ cannot normally be detected without using indirect approaches. These studies nevertheless suggest that 1H MRS may play an important role in future human studies of neuroprotective agents, particularly where brain levels of neurochemicals can be expected to reflect biological effects.

31P studies of skeletal muscle at rest showed reduced phosphocreatine to inorganic phosphate rations and reduced adenosine triphosphate/(phosphocreatine + inorganic phosphate) $)^{71,72,75}$ and reduced production of mitochondrial $\mathrm{ATP}^{75}$ during recovery from exercise in symptomatic HD, supporting a role for global mitochondrial dysfunction in HD. However, it is still unknown whether the presumed metabolic defect is simply a secondary marker of degeneration or the primary cause of neuronal death.

\section{Summary of spectroscopic studies}

Several studies done in both human and murine models of HD have demonstrated that neurochemical concentrations are altered in HD and support a role of altered energy metabolism in HD. Future studies are needed to determine whether these alterations are causative or secondary measures. Nevertheless, it is clear that spectroscopy may provide sensitive surrogate markers, especially with respect to the use of therapeutics targeted at neuroprotection or at altering metabolism.

\section{CONCLUSIONS}

Accurate and sensitive measurement of onset and progression are crucial for clinical trials of neuroprotective agents potentially able to delay onset or slow progression. Currently, the measurement of onset and progression depends on clinical instruments with high inter-rater and intrarater variability. Trials of neuroprotective treatments for HD using clinical endpoints thus require large numbers of patients and long trial durations. Newer neuroimaging techniques are providing potential markers of disease onset and progression that can be easily adapted to clinical trials.

Morphometric markers may provide the most sensitive and reliable method to determine if a drug is effective in symptomatic patients. Everything known about HD, particularly human neuropathology and data from neuroprotective therapies that are effective in HD transgenic mouse models, indicates that brain neurodegeneration corresponds to the progression of the disease and preventing or reversing neurodegeneration slows the clinical disease and is reflected in preservation of brain tissue and is detectable morphologically. Newer automated methods are being developed that will enable clinical scientists to obtain volumes of all brain structures and that may be especially applicable to clinical trials. Not only do they provide fast, reliable, and reproducible measures, but there is growing evidence that both striatal and extrastriatal degeneration have clinical importance. MRS has been used in several transgenic neuroprotective studies $^{76}$ and may play an important role in human neuroprotective studies.

Putative neuroprotective agents in HD, however, may have particular application before brain pathology becomes clinically manifest. If these agents were to be tested in clinical trials, a reliable marker of the burden and rate of progression of pathological change in the preclinical group would be needed. Functional neuroimaging with PET and fMRI may be useful for investigating the physiological underpinnings of the behavioral, cognitive, and motor changes of the disease and may be of particular relevance in studying at-risk individuals during a time before frank brain atrophy is measurable but neurons are at risk. It is still unclear whether fMRI will be useful as a biomarker to detect functional improvements in symptomatic individuals. The contribution of brain atrophy to the changes seen with PET imaging in HD has been inadequately studied and for this reason it remains uncertain whether PET will provide information regarding HD progression beyond what can be achieved with MRI.

Both cross-sectional and longitudinal studies may ben- 
efit most by the application of several neuroimaging techniques in parallel; this would provide complimentary information about the metabolic, functional, and neuroanatomic alterations that take place in the various stages of disease. Furthermore, this type of multimodal imaging may provide the most sensitive biomarker that could be used to simultaneously quantify the functional and neuroprotective properties of a drug.

\section{REFERENCES}

1. Kremer B, Goldberg P, Andrews SE et al. A worldwide study of the Huntington's disease mutation. The sensitivity and specificity of measuring CAG repeats. N Engl J Med 330:1401-1406, 1994.

2. Duyao M, Ambrose C, Myers R. Trinucleotide repeat length: instability and age of onset in Huntington's disease. Nat Genet 4:387-392, 1993.

3. Goldberg YP, Andrews SE, Theilmann J et al. Familial predisposition to recurrent mutations causing Huntington's disease: genetic risk to sibs of sporadic cases. J Med Genet 30:987-990, 1993.

4. Nance M and the American College of Medical Genetics/American Society of Human Genetics Huntington's Disease Genetic Testing Working Group. Laboratory guidelines for Huntington disease genetic testing. Am J Hum Genet 62:1243-1247, 1998.

5. The Huntington's Disease Collaborative Research Group. A novel gene containing a trinucleotide repeat that is expanded and unstable on Huntington's disease chromosomes. Cell 72:971-983, 1993.

6. Kieburtz K, MacDonald M, Shih C et al. Trinucleotide repeat length and progression of illness in Huntington's disease. $J$ Med Genet 31:872-874, 1994.

7. Feigin A, Kieburtz K, Bordwell $\mathrm{K}$ et al. Functional decline in Huntington's disease. Mov Disord 10:211-214, 1995.

8. Vonsattel JP, DiFiglia M. Huntington disease. J Neuropathol Exp Neurol 57:369-384, 1998.

9. Hersch SM. Huntington's disease: prospects for neuroprotective therapy 10 years after the discovery of the causative genetic mutation. Curr Opin Neurol 16:501-506, 2003.

10. Sapp E, Schwarz C, Chase $\mathrm{K}$ et al. Huntingtin localization in brains of normal and Huntington's disease patients. Ann Neurol 42:604612, 1997.

11. Persichetti F, Ambrose GM, Ge P et al. Normal and expanded Huntington's disease gene alleles produce distinguishable proteins due to translation across the CAG repeat. Mol Med 1:374-383, 1995.

12. Sharp AH, Loev SJ, Schilling G et al. Widespread expression of Huntington's disease gene (IT15) protein product. Neuron 14: 1065-1074, 1995.

13. Trottier Y, Devys D, Imbert $G$ et al. Cellular localization of the Huntington's disease protein and discrimination of the normal and mutated form. Nat Genet 10:104-110, 1995.

14. Zuccato C, Ciammola A, Rigamonti D et al. Loss of huntingtinmediated BDNF gene transcription in Huntington's disease. Science 293:493-498, 2001.

15. Brinkman RR, Mezci MM, Theilmann J et al. The likelihood of being affected with Huntington disease by a particular age, for a specific CAG size. Am J Hum Genet 60:1202-1210, 1997.

16. Wellington CL, Brinkman RR, O'Kusky JR et al. Toward understanding the molecular pathology of Huntington's disease. Brain Pathol 7:979-1002, 1997.

17. Davies SW, Turmaine M, Lozens BA et al. Formation of neuronal intranuclear inclusions underlies the neurological dysfunction in mice transgenic for the HD mutation. Cell 90:537-548, 1997.

18. Gutekunst CA, Li SH, Yi A et al. Nuclear and neuropil aggregates in Huntington's disease: relationship to neuropathology. J Neurosci 19:2522-2534, 1999.

19. Reddy PH, Williams M, Huang HM et al. Behavioural abnormalities and selective neuronal loss in HD transgenic mice expressing mutated full-length HD cDNA. Nat Genet 20:198-202, 1998.

20. Andreassen OA, Dedeoglu A, Ferrante RJ et al. Creatine increase survival and delays motor symptoms in a transgenic animal model of Huntington's disease. Neurobiol Dis 8:479-491, 2001.
21. Andreassen OA, Ferrante RJ, Huang HM et al. Dichloroacetate exerts therapeutic effects in transgenic mouse models in Huntington's disease. Ann Neurol 50:112-117, 2001.

22. Andreassen OA, Ferrante RJ, Dedeoglu A et al. Lipoic acid improves survival in transgenic mouse models of Huntington's disease. NeuroReport 12:3371-3373, 2001.

23. Chen M, Ona VO, Li M et al. Minocycline inhibits caspase- 1 and caspase- 3 expression and delays mortality in a transgenic mouse model of Huntington disease. Nat Med 6:797-801, 2000.

24. Dedeoglu A, Kubilus JK, Jeitner TM et al. Therapeutic effects of cystamine in a murine model of Huntington's disease. $J$ Neurosci 22:8942-8950, 2002.

25. Ferrante RJ, Kubilus JK, Lee JL et al. Histone deacetylase inhibition by sodium butyrate chemotherapy ameliorates the neurodegenerative phenotype in Huntington's disease mice. J Neurosci 23:9418-9427, 2003.

26. Huntington Study Group. Unified Huntington's disease rating scale: reliability and consistency. Mov Disord 11:1-4, 1996.

27. Alexander GE, DeLong MR, Strick PL. Parallel organization of functionally segregated circuits linking basal ganglia and cortex. Annu Rev Neurosci 9:357-381, 1986.

28. Aylward EH, Li Q, Stine OC et al. Longitudinal change in basal ganglia volume in patients with Huntington's disease. Neurology 48:394-399, 1997.

29. Rosas HD, Goodman J, Chen YI et al. Striatal volume loss in HD as measured by MRI and the influence of CAG repeat. Neurology 57:1025-1028, 2001

30. Aylward EH, Codori AM, Rosenblatt A et al. Rate of caudate atrophy in presymptomatic and symptomatic stages of Huntington's disease. Mov Disord 15:552-560, 2000.

31. Brandt J, Bylsma FW, Aylward EH et al. Impaired source memory in Huntington's disease and its relation to basal ganglia atrophy. J Clin Exp Neuropsychol 17:868-877, 1995.

32. Rosas HD, Korosheta WJ, Chen YI et al. Evidence for more widespread cerebral pathology in early HD: an MRI-based morphometric analysis. Neurology 60:1615-1620, 2003.

33. Fischl B, Salut DH, Busa E et al. Whole brain segmentation: automated labeling of neuroanatomical structures in the human brain. Neuron 33:341-355, 2002.

34. Ashburner J, Friston KJ. Why voxel-based morphometry should be used. Neuroimage 14:1238-1243, 2001.

35. Thieben MJ, Duggins AJ, Good CD et al. The distribution of structural neuropathology in pre-clinical Huntington's disease. Brain 125:1815-1828, 2002.

36. Rosas HD, Liu AK, Hersch $\mathrm{S}$ et al. Regional and progressive thinning of the cortical ribbon in Huntington's disease. Neurology 58:695-701, 2002.

37. Brandt J, Shpritz B, Codori AM et al. Neuropsychological manifestations of the genetic mutation for Huntington's disease in presymptomatic individuals. J Int Neuropsychol Soc 8:918-924, 2002.

38. Paulsen JS, Zhad H, Stout JC et al. Clinical markers of early disease in persons near onset of Huntington's disease. Neurology 57:658-662, 2001.

39. Lawrence AD, Sahakian BJ, Hodges JR et al. Executive and mnemonic functions in early Huntington's disease. Brain 119:16331645, 1996.

40. Lawrence AD, Watkins LH, Sahakian BJ et al. Visual object and visuospatial cognition in Huntington's disease: implications for information processing in corticostriatal circuits. Brain 123:13491364, 2000.

41. Jahanshahi M, Brown RG, Marsden CD. A comparative study of simple and choice reaction time in Parkinson's, Huntington's and cerebellar disease. J Neurol Neurosurg Psychiatry 56:1169-1177, 1993.

42. Witjes-Ane MN, Vegter-van der Vlis M, van Vugt JP et al. Cognitive and motor functioning in gene carriers for Huntington's disease: a baseline study. J Neuropsychiatry Clin Neurosci 15:7$16,2003$.

43. Clark VP, Lai S, Deckel AW. Altered functional MRI responses in Huntington's disease. NeuroReport 13:703-706, 2002.

44. Aron AR, Schlaghecken F, Fletcher PC et al. Inhibition of subliminally primed responses is mediated by the caudate and thala- 
mus: evidence from functional MRI and Huntington's disease. Brain 126:713-723, 2003.

45. Kunig G, Leenders KL, Sanchez-Pernaute R et al. Benzodiazepine receptor binding in Huntington's disease: [11C]flumazenil uptake measured using positron emission tomography. Ann Neurol 47: 644-648, 2000.

46. Backman L, Farde L. Dopamine and cognitive functioning: brain imaging findings in Huntinton's disease and normal aging. Scand $J$ Psychol 42:287-296, 2001.

47. Backman L, Robins-Wahlin TB, Lundin A et al. Cognitive deficits in Huntington's disease are predicted by dopaminergic PET markers and brain volumes. Brain 120:2207-2217, 1997.

48. Lawrence AD, Weeks RA, Brooks DJ et al. The relationship between striatal dopamine receptor binding and cognitive performance in Huntington's disease. Brain 121:1343-1355, 1998.

49. Pavese N, Andrews TC, Brooks DJ et al. Progressive striatal and cortical dopamine receptor dysfunction in Huntington's disease: a PET study. Brain 126:1127-1135, 2003.

50. Ghilardi M, Ghez C, Dhawan V, Moeller J, Mentis M, Nakamura $\mathrm{T}$ et al. Patterns of regional brain activation associated with different forms of motor learning. Brain Res 871:127-145, 2000.

51. Feigin A, Ghilardi MF, Ghez CP et al. Increased cingulate activation during motor sequence learning in presymptomatic Huntington's disease. Neurology 58(Suppl 3):A333-A334, 2002.

52. Carbon M, Feigin A, Ghilardi MF et al. Longitudinal changes in the relationship between striatal D2 receptor binding and brain activation in preclinical Huntington's disease. Neurology 60(Suppl 1):A247-A248, 2003.

53. Hayden MR, Martin WR, Stoessl AJ et al. Positron emission tomography in the early diagnosis of Huntington's disease. Neurology 36:888-894, 1986.

54. Kuhl DE, Phelps ME, Markham $\mathrm{CH}$ et al. Cerebral metabolism and atrophy in Huntington's disease determined by 18FDG and computed tomographic scan. Ann Neurol 12:425-434, 1982.

55. Kuwert T, Lange HW, Langen KJ et al. Cortical and subcortical glucose consumption measured by PET in patients with Huntington's disease. Brain Res 113:1405-1423, 1990

56. Young AB, Penney JB, Starosta-Rubinstein S et al. PET scan investigations of Huntington's disease: cerebral metabolic correlates of neurological features and functional decline. Ann Neurol 20:296-303, 1986.

57. Young AB, Penney JB, Starosta-Rubinstein S et al. Normal caudate glucose metabolism in persons at risk for Huntington's disease. Arch Neurol 44:254-257, 1987

58. Antonini A, Leenders KL, Spiegel R et al. Striatal glucose metabolism and dopamine D2 receptor binding in asymptomatic gene carriers and patients with Huntington's disease. Brain 119:2085$2095,1996$.

59. Hayden MR, Hewitt J, Stoessl AJ et al. The combined use of positron emission tomography and DNA polymorphisms for preclinical detection of Huntington's disease. Neurology 37:14411447, 1987.

60. Kuwert T, Ganslandt T, Jansen $\mathrm{P}$ et al. Influence of size of regions of interest on PET evaluation of caudate glucose consumption. J Comput Assist Tomogr 16:789-794, 1992.

61. Mazziotta JC, Phelps ME, Pahl JJ et al. Reduced cerebral glucose metabolism in asymptomatic subjects at risk for Huntington's disease. N Engl J Med 316:357-362, 1987.
62. Feigin A, Fukuda M, Zgaljardic D et al. Metabolic brain networks in presymptomatic Huntington's disease. Paper presented at the 15th Annual Symposia on Etiology, Pathogenesis, and Treatment of Parkinson's Disease and Other Movement Disorders, Chicago, 2001.

63. Turjanski N, Weeks R, Dolan R et al. Striatal D1 and D2 receptor binding in patients with Huntington's disease and other choreas. A PET study. Brain 118:689-696, 1995.

64. Weeks RA, Piccini P, Harding AE et al. Striatal D1 and D2 dopamine receptor loss in asymptomatic mutation carriers of Huntington's disease. Ann Neurol 40:49-54, 1996.

65. Antonini A, Leenders KL, Eidelberg D. [11C]raclopride-PET studies of the Huntington's disease rate of progression: relevance of the trinucleotide repeat length. Ann Neurol 43:253-255, 1998.

66. Andrews TC, Weeks RA, Turjanski N et al. Huntington's disease progression. PET and clinical observations. Brain 122:2353-2363, 1999.

67. Hussey D, Stewart P, Houle S et al. [C-11]raclopride striatal binding potential as a measure of Huntington's disease progression: implications for prospective neuroprotective studies. $J$ Nucl Med 39(Suppl):200P, 1998.

68. Storey E, Kowall NW, Finn SF et al. The cortical lesion of Huntington's disease: further neurochemical characterization, and reproduction of some of the histological and neurochemical features by N-methyl-D-aspartate lesions of rat cortex. Ann Neurol 32:526534, 1992.

69. Ellison DW, Beal MF, Martin JB. Amino acid neurotransmitters in postmortem human brain analyzed by high performance liquid chromatography with electrochemical detection. J Neurosci Methods 19:305-315, 1987

70. Jenkins BG, Rosas HD, Chen $4 \mathrm{C}$ et al. $1 \mathrm{H}$ NMR spectroscopy studies of Huntington's disease: correlations with CAG repeat numbers. Neurology 50:1357-1365, 1998.

71. Koroshetz WJ, Jenkins BG, Rosen BR et al. Energy metabolism defects in Huntington's disease and effects of coenzyme Q10. Ann Neurol 41:160-165, 1997.

72. Hoang TQ, Bluml S, Dubowitz DJ et al. Quantitative protondecoupled 31P MRS and 1H MRS in the evaluation of Huntington's and Parkinson's diseases. Neurology 50:1033-1040, 1998.

73. Sanchez-Pernaute R, Garcia-Segura JM, del Barrio Alba A et al. Clinical correlation of striatal 1H MRS changes in Huntington's disease. Neurology 53:806-812, 1999.

74. Ferrante RJ, Andreassen OA, Jenkins BG et al. Neuroprotective effects of creatine in a transgenic mouse model of Huntington's disease. J Neurosci 20:4389-4397, 2000.

75. Lodi R, Schapira AH, Manners D et al. Abnormal in vivo skeletal muscle energy metabolism in Huntington's disease and dentatorubropallidoluysian atrophy. Ann Neurol 48:72-76, 2000.

76. Ferrante RJ, Andreassen OA, Dedeoglu A et al. Therapeutic effects of coenzyme Q10 and remacemide in transgenic mouse models of Huntington's disease. J Neurosci 22:1592-1599, 2002.

77. Hersch SM, Ferrante RJ. Neuropathology and pathophysiology of Huntington's disease. In: Movement disorders. Neurologic principles and practice (Watts AL, Koller WC, eds), pp 503-518. Columbus, OH: McGraw-Hill, 1997. 